\title{
Beacon routing algorithm in wireless sensor networks with mobile gateway
}

\author{
Jun Xia ${ }^{1,2^{*}}$, Fei Yin ${ }^{3}$, Yun Rui ${ }^{4,2,1}$, Kai Yu ${ }^{1}$, Zhenhong $\mathrm{Li}^{3}$, Haifeng Wang ${ }^{3}$ and Zhiyong Bu ${ }^{1}$
}

\begin{abstract}
In this article, we first propose a reverse sector mechanism and an optimization division mechanism, which can provide enormous energy conservation benefits. Based on these mechanisms, an efficient sensory data collection mechanism over a cellular-WSN integrated network, named beacon routing algorithm, is proposed to spontaneously renew the local WSN topology according to the position of the UE relative to the location of the beacon cluster under an optimized network division pre-set. Different from the previous studies, beacon routing algorithm achieves the adaptive topology renewal without additional re-clustering overhead. Through performance evaluation, we can implement WSN by making a trade-off between network scale and sector division. Moreover, optimal energy efficiency can be obtained in each divided sub-network; therefore, the WSN lifetime can be increased significantly and the data collection efficiency will be enhanced. Simulation results are presented to show the performance of the proposed algorithm.
\end{abstract}

Keywords: wireless sensor network (WSN), user equipment (UE), gateway, beacon, routing

\section{Introduction}

Information sharing between different types of network entities drives the aggregation of heterogeneous networks. This kind of aggregation provides for example the possibility to exchange information between entities of a local network and a heterogeneous network. A specific scenario for a combination of capabilities of different networks for information sharing is to use a cellular network element such as a user equipment (UE) or entity as a gateway for local wireless sensor networks (WSNs). That is, elements of a cellular network and a WSN are mixed in order to easily expand the function of each network. With the natural mobility characteristic, this new type of multimode UE equipped with WSN module is similar to an enhanced type of mobile sensor node with more energy and flexibility. Since WSN can be deployed easily as less infrastructure and attention are required, the integration of cellular network and WSN seems attractive by combining the sensing part and the connection part in a flexible way, which

\footnotetext{
* Correspondence: jun.xia@mail.sim.ac.cn

'Shanghai Institute of Micro-system and Information Technology (SIMIT),

Chinese Academy of Sciences (CAS), Shanghai, China

Full list of author information is available at the end of the article
}

expands both networks' scalability and ubiquitous service applications.

In this article, we assume a type of isolated sensor network and a special sink, eNB, which solve the contradiction between the UE and the sink in terms of functionality in convergent scenario. Each UE can play a role of information collector. Since the quantity of UE is huge, it is possible to fully utilize this advantage to collect overall sensory data, enhance network transmission efficiency, and increase lifetime of sensor networks. However, as the positions of UEs are random; it is hard for a single UE to collect full information of local WSN with high efficiency. Hence, the traditional topology and routing algorithm used in WSN are hard to meet the collecting efficiency as UEs appear in different positions. For example, it has been suggested to optimize the selection of cluster heads under specific constraints. However, there are still open issues on how to solve problems related to the computation and communication overheads incurred by re-clustering caused by the stochastic nature of UEs.

WSN is subject to a unique set of resource constraints, such as finite on-board battery power and limited network communication bandwidth. It is well known that communicating 1 bit over the wireless 
medium at short ranges consumes far more energy than processing that bit. In conventional routing protocols, sensor nodes make the routing decision via collecting routing information in delivery package and following the rule of specific routing algorithm. Additional routing data exchange between sensor nodes will obviously lead to the network inefficiency and unnecessary energy waste. Thus, we endeavour on turning routing communication procedure into calculation of local sensor node and propose beacon routing algorithm to eliminate communication consumption in routing exchanging process. Sensor nodes in local network can autonomously make routing decision in an efficient way according to the polling signal containing beacon cluster identification from mobile gateway. Therefore, additional cost of routing message exchange can be saved to increase the network lifetime.

The rest of this article is organized as follows. Section 2 introduces some related studies and discusses the problem. Section 3 presents the beacon routing algorithm in more details, including reverse sector topology method, divided sector optimization, and an implementation example by adopting proposed beacon routing algorithm over a cellular-WSN integrated network. In Section 4, we present our simulation results on the proposed algorithm. Finally, we conclude this article in Section 5 .

\section{Related studies}

In a conventional WSN, sink is a local device for collecting data of the whole network. But the data collecting efficiency is low for a fixed sink unless a number of sinks are deployed, which actually divides the local network into several parts. One sink can communicate with other sinks via internet for the purpose of sharing information. Perhaps the most profound difference between sensor networks and other types of networks, wired or wireless, comes from the fact that the classical separation of address and content in a packet is no longer viable in the sensor net setting $[1,2]$.

In WSNs, dense networks of distributed communicating sensors can improve signal-to-noise ratio by reducing average distances from sensors to the source of signal, or target. Increased energy efficiency in communications is enabled by the multi-hop topology of the network [3]. A solution to the localization problem can specify a set of sensor nodes on a path that gather and combine data as they route the result back to the querying node. Conventional protocols, for example flooding or gossiping-based routing [4], waste energy and bandwidth to transfer extra and unnecessary copies of data in overlapping areas. In some hierarchical topology based sensor networks, each cluster elects a cluster-head node, and routing is done only among the cluster-heads (the remaining nodes always route packets through their cluster-heads) [5]. This is advantageous for a variety of reasons, including the possibility of using simpler communication protocols within a cluster, recycling of resources (such as frequency assignments) among disjoint clusters, and saving power. The focus of the existing literature has mostly concentrated on optimizing the choices of cluster heads to meet some constraints. However, fewer studies have been devoted on how to alleviate the computation and communication overheads caused by re-clustering that spurs the development of a gateway node for a WSN.

Mobile sink WSN (MSWSN) is similar to the scenario described in Section 1, since UEs actually serve as mobile sink with random mobility in local WSN. No network information is required as the decision of a sink to decide next sojourn position is random. The movement pattern of a sink does not depend upon network conditions, which may not lead to optimal network lifetimes. Random mobility requires continuous sink position updates and route reconstruction, which will increase the transceiver burden of each sensor nodes.

Flooding is an easy routing protocol $[6,7]$, in which each node only needs to broadcast the newly received message to its neighbouring nodes, not requiring topology records or large amount of analyses. This routing protocol is reliable. Regardless of any changes in the network, data can be transmitted to the sink node in the way of flooding as long as the path between the source node and the sink node exists. But flooding consumes much energy, because for each data packet, all nodes in the broadcast domain will receive the packet and forward it to its neighbours. The large amount of power required by flooding causes a prohibitively short network lifetime, which makes it difficult to apply basic flooding protocol to real WSNs.

Gossiping [4] is an alternative to the classic flooding approach that uses randomization to conserve energy. Instead of indiscriminately forwarding data to all its neighbours, a gossiping node only forwards data on to one randomly selected neighbour. If a gossiping node receives data from a given neighbour, it can forward data back to that neighbour if it randomly selects that neighbour. Whenever data travel to a node with high degree in a classic flooding network, more copies of the data start floating around the network. At some point, however, these copies may end up imploding. Gossiping avoids such implosion because it only makes one copy of each message at any node. The fewer copies it made, the lower the likelihood that any of these copies will ever implode. Although gossiping largely avoids implosion, it does not solve the overlap problem as a gossiping node receives data from a given neighbour, it can 
forward data back to that neighbour if it randomly selects that neighbour. In addition, the gossiping approach does not solve the sink mobility problem.

Data centric routing mechanisms such as directed diffusion [8] conduct routing is similar to AODV in ad hoc network, routing request and reply are necessary during the period of path establishment. Path discovery depends on the exchange of inquiring packet among different nodes in network. This kind of routing mechanism performs better than simple flooding algorithms, but it still relies on basic flooding mechanism during the period of path discovery. Some protocols consider the classical flooding as the basic approach in MSWSN for initializing network and packet delivery. ART Protocol [9] proposed an adaptive reversal tree-based algorithm. A tree directed towards sink assigned temporary root node is created. This is achieved through initial flooding. Source nodes use this tree to direct data reports towards root node which then delivers data to the sink. ART efficient path repair mechanism reduces sink's communication overhead but may result in suboptimal source to sink path. ALURP and LURP are proposed in [10] that include a geographic data dissemination approach. In LURP, sink initially floods its location information plus a virtual circular area (VC) centred on its current position. Then, as long as sink mobility is confined in VC, local broadcast is performed. Exit from VC requires global flooding. Nodes outside $\mathrm{VC}$ use geographic routing to route data towards sink. Once packet arrives inside $\mathrm{VC}$, shortest path routing is used on sink updated paths. In ALURP, radius of VC is adapted according to the mobility of the sink. This reduces local broadcast cost. In addition, numbers of global flooding requirements are also reduced as VC size can be dynamically changed.

Complete Graph-based Clustering Algorithm (CGCA) [11] is proposed in a densely deployed sensor network. CGCA divides the network into a few complete graphs; each complete graph independently becomes a cluster. CGCA introduces mobile nodes as gateways and verifies its efficiency in lengthening lifetime of WSN. However, the algorithm imposes some restriction on the behaviour of the mobile gateway, such as roaming around the deployment region, which is unnecessary as more efficient and flexible schemes can be proposed.

The discussion about integration of heterogeneous network is very prevalent, but the content about WSN and cellular is scarce. The routing algorithms and topology control methods described above may be used to solve the problems in each specific scenario, but they cannot be applied in the scenario we suggested in Section 1 straightforwardly. The traditional sink as well as mobile sink are different from UE in terms of distribution density, transmission capability, mobility randomness, etc.

\section{Beacon cluster routing algorithm}

Sensor networks extend the existing internet deep into the physical environment. A WSN for a local sensor network may consist of spatially distributed autonomous sensors which are configured to monitor different parameters, such as physical or environmental conditions like temperature, sound, pressure, movements, concentrations of specific elements in the air, etc. Each node equipped with one or more sensing devices such as acoustic microphone arrays, video or still cameras, infrared, seismic, or magnetic sensors. The sensor nodes are further configured to cooperatively pass data through a network to a main location which is also referred to as sink. Also bi-directional communication between the sensor nodes and the sink is possible in order to enable a control of the sensors. WSNs are used, for example, in many industrial and consumer applications, such as industrial process monitoring and control, machine health monitoring, environment and habitat monitoring, healthcare applications, traffic control, etc. The nodes of the WSN may comprise sensor nodes (SNs) and one or more cluster head $(\mathrm{CH})$ nodes. Cluster-head nodes are used as managing nodes when a WSN is divided into one or more clusters containing plural sensor nodes and one cluster head node. The sensor nodes are connected to one (or sometimes several) other sensor nodes, wherein their data are forwarded to a respective cluster head which transmit the aggregated information to the sink. A sensor node has typically several parts: a transceiver with an antenna or connection to an antenna, a microcontroller, an electronic circuit for interfacing with the sensors and an energy source, e.g. a battery or an embedded form of energy harvesting. The topology of a WSN can vary from a simple star shape to a multi-hop mesh network.

The largest design constraint, however, is the limited energy budget of a sensor node together with the requirement of long network runtimes. For instance, having a node continuously powered on drains an AA battery of $3000 \mathrm{mAh}$ in about 4 days [12], which is well below the typically required decade of operation. On the other extreme, even if a node is switched off all the time, inherent current leakages in the battery limits the battery lifetime to $10-15$ years in dependency of the operating temperature. This renders network lifetime maximization with battery-powered nodes useless if the lifespan goes beyond this number [13]. In typical sensor applications, the energy consumption is dominated by the node's radio consumption. A prominent example of the current non-optimized WSN deployment is that the 
start-up alone costs the network a third of its battery power $[13,14]$. Based on these constraints for WSN, high efficient network implementation scheme and data collection under power saving mode is essential for practical applications.

\subsection{Assumptions}

Generally, the coverage of WSN can be approximately considered as a circular region. For energy efficiency consideration, local sink is inclined to be implemented in the middle of the coverage region. Thus, we can divide the circular region into sectors by slicing the region from the centre of the circle. The following analysis is based on this basic geometry. In this model, we ignore all radio propagation effects such as obstacle or multipath interference, anisotropy or asymmetry of transmission, etc. We assume all nodes have the same transmission range which is set to be equal to $r$ or rescaled if necessary. To make it easy for analysis, we further assume the following:

- Each sensor node is homogeneous. Local sensor network is implemented as flat with a unique sink node.

- Index number $i$ denotes the layer sequence of the divided sector layer; similarly, index number $j$ denotes the block sequence of the divided mini sector which represents the divided piece of each layer in the sector.

- The radius of the sector is $R$, and the transmission range of each sensor node is $r$.

- The primary energy consumption of each transmission or reception is approximate to the same basic energy unit $E_{\mathrm{b}}$.

- The vertex angle of sector is set as $\beta$; and density of sensor node distributed in the sector is represented by $\rho$.

- Energy consumption of the primary mini sector is specified as $E_{i j}$.

- Chain type network is considered as the basic network model in the following analysis, in which energy consumption can be represented as $E_{\text {chain }}$, each sensor node in the chain consumes $E_{\text {sent }}$ energy units to transmit data and $E_{\text {reactive }}$ energy units to receive data.

- Energy consumption of the whole sector is specified as $E_{\mathrm{sec}}$, and for the reverse topology sector case is $E_{\mathrm{rsec}}$.

- Energy consumption of network flow inside the sector layer is denoted as $E_{\text {intra }}$, which represents the whole energy consumption of total mini sectors in the same layer.

- Energy consumption of network flow between the sector layers is denoted as $E_{\text {inter, }}$ which represents the energy consumption between each divided layers.

\subsection{Reverse sector topology}

First, we consider a basic chain type network. As illustrated in Figure 1a, there are two source nodes, one

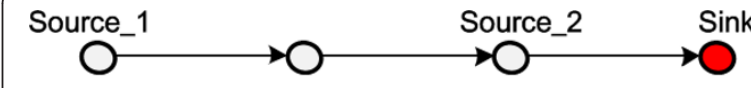

(a)

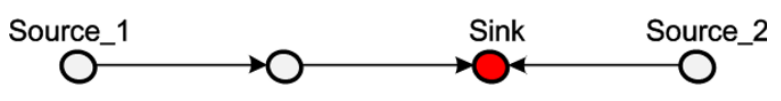

(b)

Figure 1 Chain type network.

relay node and one sink on the chain link. Each source node has its own sensory data for dissemination, and the relay node only forwards the data packet originated from source nodes. In terms of source node \#2, it also plays the role of relay node. But it can combine the relaying packet with its own packet, and send them together. Thus, the energy consumption during the whole transmission process can be represented as below:

$$
E_{\text {chain }}=3 E_{\text {sent }}+3 E_{\text {receive }}
$$

To make the analysis easier, we assume the transmission power is equal to the receiving power (denoted as $\left.E_{\mathrm{b}}\right)$ at each node. Then the total energy consumption is $6 E_{\mathrm{b}}$. If we switch the position of source node \#2 and the sink, then recalculate the energy consumption, it is straightforward to find that the total energy consumption is the same as above.

Through this simple process, we can find that simply switching the position of sink and source as illustrated in Figure $1 b$, does not impact the network energy conservation. But if the energy consumption at sink node side is ignored, which means the sink node is an energy sufficient device, the result will be totally different. Under this assumption, $E_{\text {chain }}$ in Equation (1) can be modified to $3 E_{\text {sent }}+2 E_{\text {receive. }}$ Similarly, $E_{\text {chain }}$ in switching situation can be modified to $3 E_{\text {sent }}+E_{\text {receive }}$. Thus, we can find that under the assumption of energy free sink device, simply changing the location and the network flow without modify the topology can enhance the network energy efficiency. This also illustrates that the powerful sink node shall be placed closer to the middle position of the network for energy efficiency consideration.

Obviously, decreasing the number of relay hops in the network can efficiently lower the network transmission consumption, which is a well-known principle in network optimization. For general analysis, the total energy consumption of $N$ nodes in a chain type network can be 
concluded as a transmission with $N-1$ hops and reception energy consumption is

$$
E_{\text {chain }}=(N-1)\left(E_{\text {sent }}+E_{\text {receive }}\right)
$$

As described in [15], transmit power consumption of sensor node is similar to the receive power consumption. Thus, we can still use the prior assumption that the basic energy consumption unit is $E_{\mathrm{b}}$. According to this assumption, the total energy consumption of the chain topology can be calculated as

$$
E_{\text {chain }}=2 E_{b}(N-1)
$$

Based on this, each sector in the sector topology is part of the round region and covered by a sink. As shown in Figures 2 and 3, a sector is divided into $\lceil R / r\rceil$ layers according to one-hop transmission range. Layer closer to the sink possesses the higher forwarding burden, which is called high layer. The layer far from the sink can be defined as lower layer as its forwarding frequency is lower.

As illustrated in Figure 2, the sink node is closer to the vertex of sector. There is a high probability of intermediate sensor nodes to keep active status for forwarding data packets from its child nodes. Intermediate nodes that are closer to the sink consume more energy for forwarding even itself has no sensory data needs to report. In other words, if fewer hops exist in one path from lower layer node to sink or less number of lower layer nodes in the path, forwarding frequency of intermediate nodes can be significant lower, as illustrated in Figure 3. In this case, the positions of the sink and source are reversed. The maximum hop in reverse sector can be estimated as max $\{\lceil\beta R / 2 r\rceil-1,\lceil R / r\rceil-1\}$. Even $\beta / 2 \leq 1$, the maximum number of hops in reverse sector

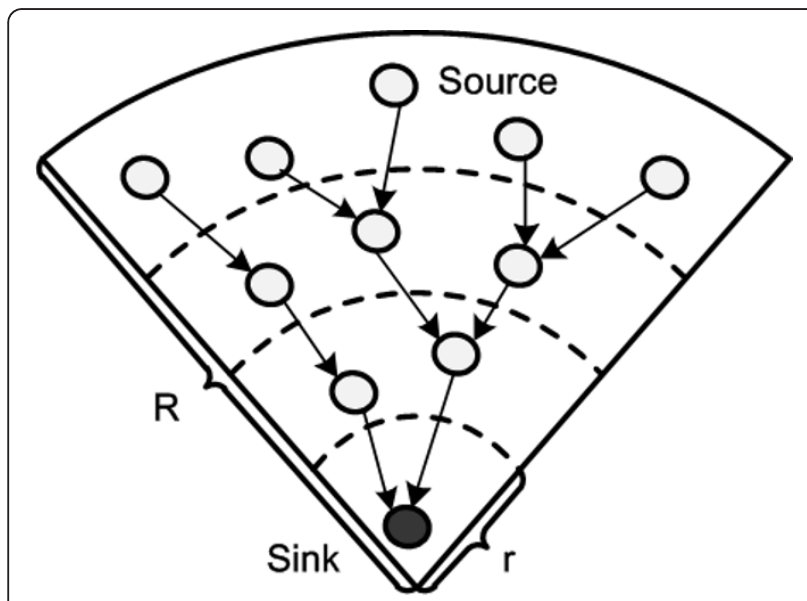

Figure 2 sector topology

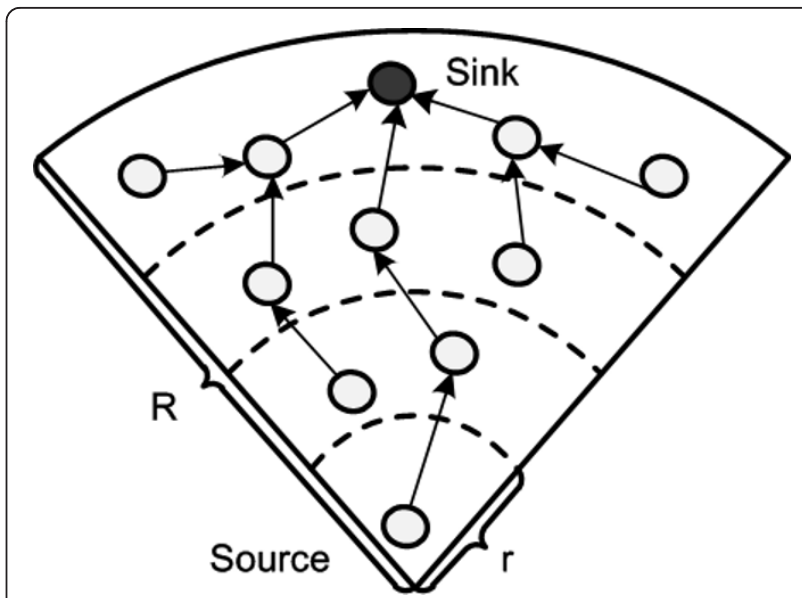

Figure 3 Reverse sector topology.

topology is still lower than in the normal case, thus the energy burden of the whole sector in reverse sector topology is also lower than in the normal case.

Below we try to analyse the sector topology energy model. Assume the sensor networks located in the sector are referred as the complete binary tree topology, it is easy to find there are $2^{i-1}$ chain links flowing to the sink in layer $i$. Each chain flow possesses $(i-1)$ hops to the sink. Substitute the parameter in Equation (3); we can get the total energy consumption of sector shown in Figure 2 as

$$
\begin{aligned}
E_{\text {sec }} & =\sum_{i=1}^{\lceil R / r\rceil} 2 E_{b} \cdot 2^{i-1}(i-1) \\
& =E_{b}\left[(\lceil R / r\rceil-2) 2^{\lceil R / r\rceil+1}+4\right]
\end{aligned}
$$

Similarly, for the reverse sector topology, we can also represent the network energy consumption with basic chain flow energy model. Then the flow of each layer to the sink may consist of two types of chain flows, which are the inter layer flows and intra layer flows. The inter layer flows include all the vertical chains in Figure 3. To make it easy for calculation, we can stochastically pick a single sensor in each layer to consist the inter layer flow. Thus, the intra layer flows consist of all the chain flows that exist in each single layer and each node prefer transmit data to the node nearby in the same layer. Here the total energy consumption can be estimated as

$$
\begin{aligned}
& E_{\text {see }}=E_{\text {inter }}+E_{\text {intra }} \\
& =\left\{\sum_{i=1}^{[R / 7]} 2 E_{b}(i-1)\right\}_{\text {inter }}+\left\{\sum_{i=1}^{[R / 7\rceil} \sum_{j=1}^{[(i-1 / 2) \beta\rceil} 2 E_{b} \cdot(j-1) \cdot \frac{2^{i-1}}{\lceil(i-1 / 2) \beta\rceil}\right\}_{\text {intra }} \\
& =E_{b} \cdot \sum_{i=1}^{[R / r]}\left\{2(i-1)+2^{i-1} \cdot[[(i-1 / 2) \beta]-1]\right\} \\
& \leq E_{\mathrm{b}} \cdot\left\{{ }^{[R / r\rceil}\left(\lceil R / \tau\rceil \beta-\frac{3}{2} \beta\right)+\lceil R / r\rceil^{2}-\lceil R / r\rceil+\frac{1}{2} \beta+1\right\}
\end{aligned}
$$


The energy consumption in normal case and reverse case can be compared as follows:

$$
\begin{aligned}
& E_{\text {gain }}=E_{\text {sec }}-E_{\text {sec }} \\
& \geq E_{\mathrm{b}}\left[([R / r]-2) 2^{[R / \tau]+1}+4\right]-E_{\mathrm{b}} \cdot\left\{2^{[R / / 7}\left([R / r] \beta-\frac{3}{2} \beta\right)+([R / r])^{2}-\lceil R / r\rceil+\frac{1}{2} \beta+1\right\} \\
& =E_{\mathrm{b}} \cdot\left\{2^{[R / r]} \cdot\left(2[R / r]-\beta\lceil R / r\rceil+\frac{3}{2} \beta-4\right)-([R / r])^{2}+\lceil R / r]-\frac{1}{2} \beta+3\right\}
\end{aligned}
$$

We substitute $\lceil R / r\rceil$ as $M$, which can be defined as network scale here. Thus, inequality (6) can be rewrite as

$$
E_{\text {gain }} \geq E_{\mathrm{b}} \cdot\left\{2^{M} \cdot\left(2 M-\beta M+\frac{3}{2} \beta-4\right)-M^{2}+M-\frac{1}{2} \beta+3\right\}
$$

From inequality (7), it is easy to see that the inequality is consisted by an exponential part and a parabola part. The exponential part actually determines the $E_{\text {gain }}$, with the increasing of parameter $M . E_{\text {gain }}$ tends to be a monotonic increase function with the growth of $M$. As a conclusion, through simple network flow reverse operation, energy cost reservation can be achieved especially in large scale network.

\subsection{Divided sector optimization}

A well-known wireless network capacity obtained by Gupta and Kumar states that the per node throughput scales as $1 / \sqrt{n}$. In other words, it goes to zeros as the number of nodes $N$ in a wireless ad hoc network increases [16]. As the number of nodes increases, every node spends almost all of its time forwarding packets of the other nodes. On the basis of this theory, the scale of local sensor network needs to be maintained in small size in order to guarantee the throughput. On the other hand, if we divide the local sensor network into several parts, the scale of the network is reduced while the throughput of each part is enhanced accordingly. This idea drives us to optimize the division process.

In binary tree topology analysis, we find that it is possible to optimize the local sensor network energy efficiency by making a trade-off between parameters $M$ and $\beta$. Similar to the ordinary sector performance, node density is ignored which may deteriorate network performance gradually in the reverse sector topology. For this reason, we analyse the general tree topology situation without specific topology. Assuming the sensor node is scattered in the sector region uniformly distributed. The density of the sensor nodes within each square meter is $\rho$. Hence, number of nodes in each layer is

$$
N=\frac{(2 i-1)}{2} \beta \rho r^{2}
$$

Similar to the above case in binary tree topology, network flows in the general sector topology can still be considered as the combination of chain flow originated from each node to the sink. Total energy consumption of the sector can be calculated as

$$
\begin{aligned}
E_{\mathrm{sec}} & =\sum_{i=1}^{\lceil R / r\rceil} 2 E_{\mathrm{b}} \beta \rho r^{2} \frac{(2 i-1)}{2}(i-1) \\
& =\frac{E_{\mathrm{b}} \beta \rho r^{2}}{6}\lceil R / r\rceil(4\lceil R / r\rceil+1)(\lceil R / r\rceil-1)
\end{aligned}
$$

In Equation (9), sector energy consumption $E_{\text {sec }}$ varies with vertex angle $\beta$ and the nodes density $\rho$. Comparing with Equation (4), the energy consumption of the sector type network is degraded from the exponential growth to the power function growth, which is reasonable in practical scenario. As in the hierarchical network, depth of the network should not be set too large for sensor node lifetime consideration. With the increase of network depth, possibility of intermediate nodes out of order rises accordingly. Especially in large scale sensor network, exponential model of energy consumption is obviously unacceptable in practical implementation.

After obtaining the energy consumption in the normal case, we need to estimate the expenditure in reverse case. Similarly, network flows can be categorized as inter sector and intra sector parts, which possess the same meaning as in the binary tree topology analysis, i.e.

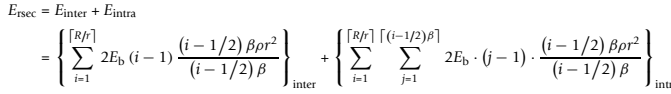

$$
\begin{aligned}
& =\sum_{i=1}^{\lceil R / \gamma]} 2 E_{\mathrm{b}} \rho r^{2}(i-1)+\sum_{i=1}^{[R / \gamma]} \sum_{j=1}^{(i-1 / 2) \beta]} 2 E_{\mathrm{b}} \cdot(j-1) \cdot \rho r^{2} \\
& =2 E_{\mathrm{b}} \rho r^{2} \cdot \sum_{i=1}^{\lceil R / \gamma]}\left[(i-1)+\frac{1}{2}([(i-1 / 2) \beta\rceil-1)\lceil(i-1 / 2) \beta\rceil\right] \\
& \leq 2 E_{\mathrm{b}} \rho r^{2} \cdot \sum_{i=1}^{[R / T]}\left\{(i-1)+\frac{1}{2}[(i-1 / 2) \beta+1](i-1 / 2) \beta\right\} \\
& =E_{\mathrm{b}} \rho r^{2}\lceil R / r\rceil\left\{\frac{1}{3} \beta^{2}\lceil R / r\rceil^{2}+\left(\frac{1}{2} \beta+1\right)\lceil R / r\rceil-\frac{1}{12} \beta^{2}-1\right\}
\end{aligned}
$$

In Equation (10), if we consider $\lceil R / r\rceil$ as an independent variable of function $E_{\mathrm{rsec}}$, the core part of the above function can be approximated as a quadratic function. Comparing the energy consumption of normal case and reverse case, the energy saved after the reverse process can be obtained as

$$
\begin{aligned}
E_{\text {gain }} & =E_{\text {sec }}-E_{\text {rsec }} \\
& \geq \frac{E_{\mathrm{b}} \beta \rho r^{2}}{6}\lceil R / r\rceil(4\lceil R / r\rceil+1)(\lceil R / r\rceil-1) \\
& -E_{\mathrm{b}} \rho r^{2}\lceil R / r\rceil\left\{\frac{1}{3} \beta^{2}\lceil R / r\rceil^{2}+\left(\frac{1}{2} \beta+1\right)\lceil R / r\rceil-\frac{1}{12} \beta^{2}-1\right\} \\
& =E_{\mathrm{b}} \rho r^{2}\lceil R / r\rceil\left\{\frac{1}{3} \beta(2-\beta)\lceil R / r\rceil^{2}-(1+\beta)\lceil R / r\rceil+\frac{1}{12} \beta^{2}-\frac{1}{6} \beta+1\right\}
\end{aligned}
$$

Rewritten the inequality (11) into inequality (12) based on parameter $\beta$, we get

$$
E_{\text {gain }} \geq E_{\mathrm{b}} \rho r^{2}\lceil R / r\rceil\left\{\left(\frac{1}{12}-\frac{1}{3}\lceil R / r\rceil^{2}\right) \beta^{2}+\left(\frac{2}{3}\lceil R / r\rceil^{2}-\lceil R / r\rceil-\frac{1}{6}\right) \beta-\lceil R / r\rceil+1\right\}
$$

In order to find the maximum value of $E_{\text {gain }}$, we take the derivative of $E_{\text {gain }}$ with respect to $\beta$, and impose the derivation equal to 0 


$$
\frac{d E_{\text {gain }}}{d \beta}=\left(\frac{1}{6}-\frac{2}{3}\lceil R / r\rceil^{2}\right) \beta+\frac{2}{3}\lceil R / r\rceil^{2}-\lceil R / r\rceil-\frac{1}{6}=0
$$

Obviously, we get $\beta$ when $E_{\text {max_gain }}$ achieves the maximum

$$
\beta=1-\frac{6\lceil R / r\rceil}{\left(4\lceil R / r\rceil^{2}-1\right)}
$$

If $\lceil R / r\rceil$ grows large enough, then we can get the limitation of $\beta$ :

$$
\lim _{\lceil R / r\rceil \rightarrow+\infty} \beta=1
$$

According to the optimized parameter $\beta$, we can get the max energy gain as

$$
E_{\text {max gain }}=E_{\mathrm{b}} \rho r^{2}\lceil R / r\rceil\left(\frac{1}{3}\lceil R / r\rceil^{2}-2\lceil R / r\rceil+\frac{11}{12}\right)
$$

From the above results, the maximum energy gain can be achieved depending on the vertex angle of the divided sector in the circular region. But in practical applications, implementation complexity and expenditure cannot be ignored, which may constraint the number of divisions. In these practical cases, we may have to give up the optimal scheme, choose the sub-optimal division number. By dividing Equation (12) with Equation (9), we can get the energy gain efficient function as follows.

$$
E_{\text {efficient }}=\frac{E_{\text {gain }}}{E_{\text {sec }}} \geq \frac{2 \beta(2-\beta)\lceil R / r]^{2}-6(1+\beta)\lceil R / r\rceil+\frac{1}{2} \beta^{2}-\beta+6}{\beta(4\lceil R / r\rceil+1)(\lceil R / r\rceil-1)}
$$

If we consider $\lceil R / r\rceil$ as the variable in Equation (17), we can get the limitation of $E_{\text {efficient }}$ :

$$
\lim _{\lceil R / r\rceil \rightarrow+\infty} E_{\text {efficient }}=1-\frac{\beta}{2}
$$

Equation (18) shows that $E_{\text {efficient }}$ increases when $\beta$ decreases. In reality, when the scale of the network grows, smaller $\beta$ keeps the network more efficient in terms of energy. In the case that the value of $\beta$ is low, which means the reverse section operation will bring more energy conservation for small sector network, the maximum gain of the reverse section cannot be achieved. The trade-off between energy efficiency and energy gain needs to be carefully considered during the practical implementation.

\subsection{Beacon routing algorithm implementation}

Since we can pre-implement local sensor network according to the optimal division described above, beacon routing algorithm based on these divided sectors can be achieved. The beacon routing algorithm is a method of pre-computation based on divided sector model to aid the routing.

We assume the pre-deployed WSN network is segmented into multiple sectors without sink and each group has one cluster head to minimize the power consumption and increase the WSN lifetime, i.e., most of nodes are in sleep mode and only the cluster heads beacons regularly for the purpose of providing basic WSN information. Each cluster with its cluster head is identified by the unique id (namely the beacon cluster id) and each cluster is named as beacon cluster.

We make some important assumptions as follows:

- The topology of sensor network is semi-static.

- UE as mobile gateway for collecting local sensor network data is also semi-static during the data collecting period.

- UE possess the capability to directly notify every sensor node its up-to-date information via broadcasting.

- Cluster division and cluster-head selection are predefined during the network implementation period.

Once a UE starts to collect WSN information and access the WSN beacon cluster head, it senses the beacon transmission by beacon cluster head in WSN, collects the synchronization information, signal strength as well as local beacon cluster information. Then the UEs report the accessing request to the $\mathrm{eNB}$ who selects the UE gateway among UEs nearby the beacon cluster and allocate broadcasting sequence of each UE. The selected UEs trigger the cluster head and awake all WSN nodes in the network, broadcast topology update notification to inform sensor nodes to change their network flow direction. Before a UE updates the location information stored in sensor nodes, it should activate sensor nodes via preamble sample technique [17-19]. Each sensor node with pre-defined routing table changes the network flow direction correspondingly. This ensures that the whole WSN information can be collected by the mobile gateway in an efficient way.

Initialization configuration of beacon cluster is essential for the local sensor network. Once an eNB or UE needs to collect WSN information, some UEs will be selected to serve as mobile gateways. Figure 4 shows a WSN segmented into six groups with six cluster heads as an example.

Cluster head of each beacon cluster initializes a flooding procedure to pre-configure the routing table of each sensor node in the network. Intermediate nodes receive the flooding packet and modify the hop count by adding the current hop. According to the reception sequence of the flooding packet from each beacon cluster, intermediate nodes maintain a routing table with the parameters consisted of beacon cluster number, accumulated hop 


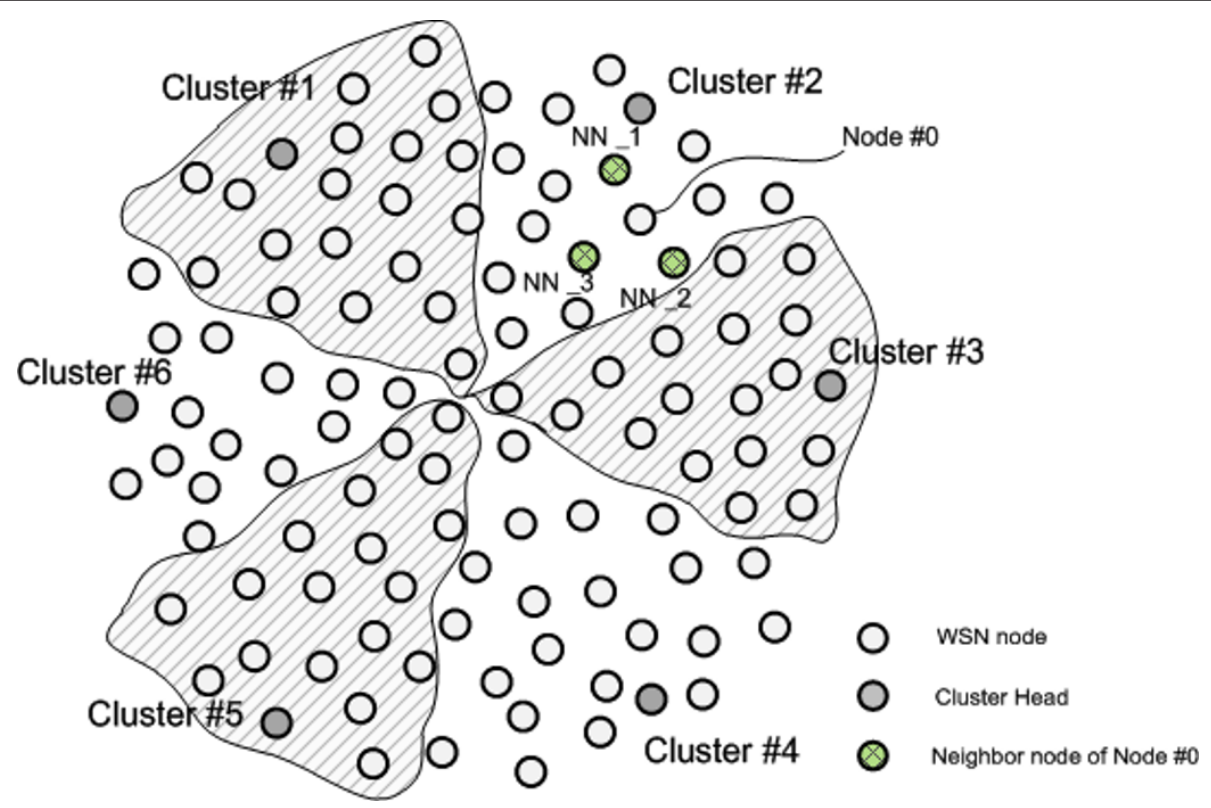

Figure 4 Optimal segmented beacon cluster.

number and the responding relay node towards the specified beacon cluster. Routing table of node \#0 in Figure 4 is illustrated in Table 1.

Table 1 is a common routing table with the same function as traditional routing methods. In practical scenarios, several UEs can appear near different cluster head of beacon cluster simultaneously. Obviously, this simple routing table cannot deal with the situation anymore. Some modification is necessary for the initialization routing table. Thus, we present a conversion algorithm for converting the rough routing table to a routing table based on beacon cluster combination.

After the convert process, each node in the sensor network can get its own routing table according to the beacon cluster. Thus, each sensor nodes have its predefined routing table with different network flow directions for different destination. Sensor nodes maintain in the idle mode, and only the cluster head beacons regularly for the purpose of minimizing power consumption. Using the conversion algorithm, routing table of node

Table 1 Routing table initialization at node \#0 after flooding procedure

\begin{tabular}{lll}
\hline Beacon cluster ID & Last hop & Hop count \\
\hline Cluster \#1 & NN \#3 & 6 \\
Cluster \#2 & NN \#1 & 2 \\
Cluster \#3 & NN \#2 & 4 \\
Cluster \#4 & NN \#2 & 8 \\
Cluster \#5 & NN \#3 & 11 \\
Cluster \#6 & NN \#3 & 9 \\
\hline
\end{tabular}

$\# 0$ is calculated and shown in Figure 4. From the new routing table, each sensor node can decide the dissemination direction according to the priority and set of beacon cluster activated by the UE. This means each sensor nodes can make the routing decision independently.

In order to explain the beacon routing algorithm explicitly, we illustrate three basic scenarios according to the scenario shown in Figure 4. Here, we call the UE as a mobile gateway in local WSN. In Figure 5a, only one mobile gateway accesses beacon cluster \#2 for data collection. After the gateway obtains the permission from eNB to collect local network information, it broadcasts a signal to awake the sleeping sensor nodes. The awakened sensor nodes keep listening for the following broadcasting message about beacon cluster ID from the gateway. As illustrated in Figure $5 \mathrm{a}$, node $\# 0$ receives notification about beacon cluster $\# 2$, and then it checks its local routing table as shown in Table 2. According to the local routing table of node $\# 0$, with the highest priority, node \#0 selects node \#1 as its next hop and starts the data dissemination.

After data collection, mobile gateway depicted in Figure 5 a leaves the sensor fields. Meanwhile, four new mobile gateways appear to collect data. In Figure 5b, there are four gateways in network; node \#0 receives ID notification about beacon cluster \#1, beacon cluster \#3, beacon cluster \#5 and beacon cluster \#6 from mobile gateway. It then checks the routing table in Table 2. As the beacon notification of cluster \#2 has expired, the second priority in Table 2 (i.e. priority 4) guides node $\# 0$ to change its traffic flow direction to node \#2. In this 


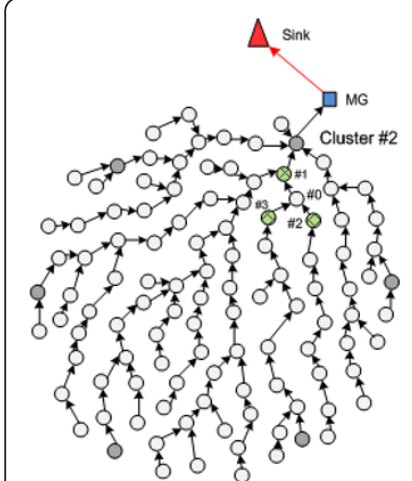

(a) one mobile gateway

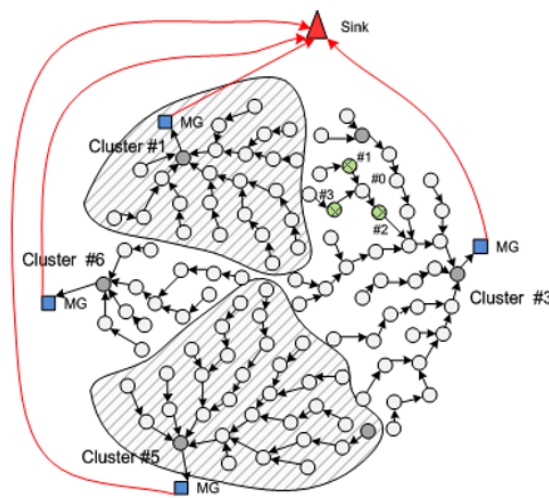

(b) four mobile gateways

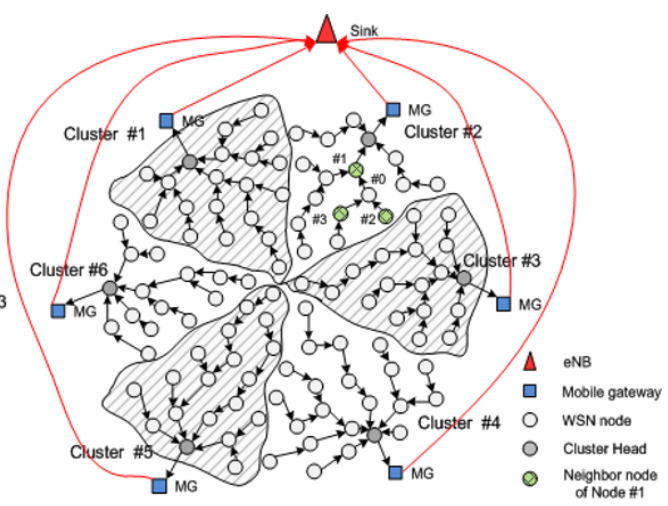

(c) six mobile gateways

Figure 5 Beacon cluster routing with multiple mobile gateway.

routing switching process, routing information interaction among sensor nodes is unnecessary since each sensor node can choose the efficient dissemination direction independently according to the beacon signal from mobile gateway. In Figure 5c, each beacon cluster head has a corresponding gateway, node \#0 selects node $\# 1$ as its next hop according the cluster set with highest priority in Table 2. Furthermore, data collection efficiency is enhanced with more mobile gateways.

Based on the implementation procedure described above, information collection process in the convergent network combined with cellular and WSN can be described as follows: Once an eNB wants to collect specific WSN information, it broadcasts its request with the related WSN identity to the UEs nearby. The UEs equipped with WSN module will overhear the periodic beacon signals from cluster heads in WSN. Then the UEs extract cluster ID from beacon signal and report to the eNB. The eNB selects and notifies proper UEs as mobile gateways for data collection. Those selected UEs awake all the sensor nodes in local network via broadcasting. During the broadcasting period, the selected UEs as mobile gateways broadcast the beacon cluster number to all the sensor nodes. The sensor nodes automatically start data dissemination process in an efficient network flow direction according to the pre-defined routing table as depicted in Table 2. Beacon routing algorithm guides each sensor

Table 2 Routing table according to the combination of beacon cluster id

\begin{tabular}{lll}
\hline Priority & $\begin{array}{l}\text { Optional beacon cluster id } \\
\text { notification }\end{array}$ & $\begin{array}{l}\text { Traffic flow direction } \\
\text { decision }\end{array}$ \\
\hline 2 & $\{$ Cluster \#2\} & NN \#1 \\
4 & $\{$ Cluster \#3, Cluster \#4\} & NN \#2 \\
6 & $\{$ Cluster \#1, Cluster \#5, Cluster \#6\} & NN \#3 \\
\hline
\end{tabular}

nodes to select the most efficient path to the current mobile gateways.

\section{Performance evaluation}

In this section, we present the simulation results of reverse sector theory, optimization division theory and beacon cluster routing algorithm. First, we set some basic parameters as follows: $E_{\mathrm{b}}$ is $0.36 \mathrm{~J}$, density of sensor nodes in mini-sector $\rho$ is 0.3 per square meters, and the transmission range of each sensor nodes is fixed to $5 \mathrm{~m}$. According to the analysis in Section 3, we can obtain the results of reverse sector theory through simulations. As demonstrated in Figure 6, it is clearly shown that with the growth of $M$; the curvature of the $E_{\text {gain }}$ curve depends mainly on the vertex angle parameter. Under the same configured $M$, decreasing $\beta$ can simply enhance the sector type network energy consumption performance by reversing the tree topology.

Based on the simulation results shown above, we study the relationship between parameter $M$ and $\beta$. The value of $M$ is consisted of the sector radius $(R)$ and the sensor node transmission range $(r)$. In general, sensor node transmission range is limited. Hence, we can regard $M$ as mainly depends on $R$. It decides the coverage region of the local sensor network. If the value of the sector radius is small, with the growing of $\beta$, reverse topology may not achieve energy saving effect as shown in Figure 7. On the other hand, when the network scale is relatively small, large value of $\beta$ cannot be acceptable for the cases such as $\beta=1.4$ and $\beta=1.8$ as shown in Figure 7 . However, with large sector vertex angle, enlarging the coverage of sensor network via increasing $R$, reverse topology still outperform the conventional method, which is the only way to optimize the network in this scenario. Note that choosing a proper value of $\beta$ can achieve more energy gain with same network scale.

In Figures 8 and 9, with the growth of network scale, energy efficiency ranks with the vertex angle of divided 


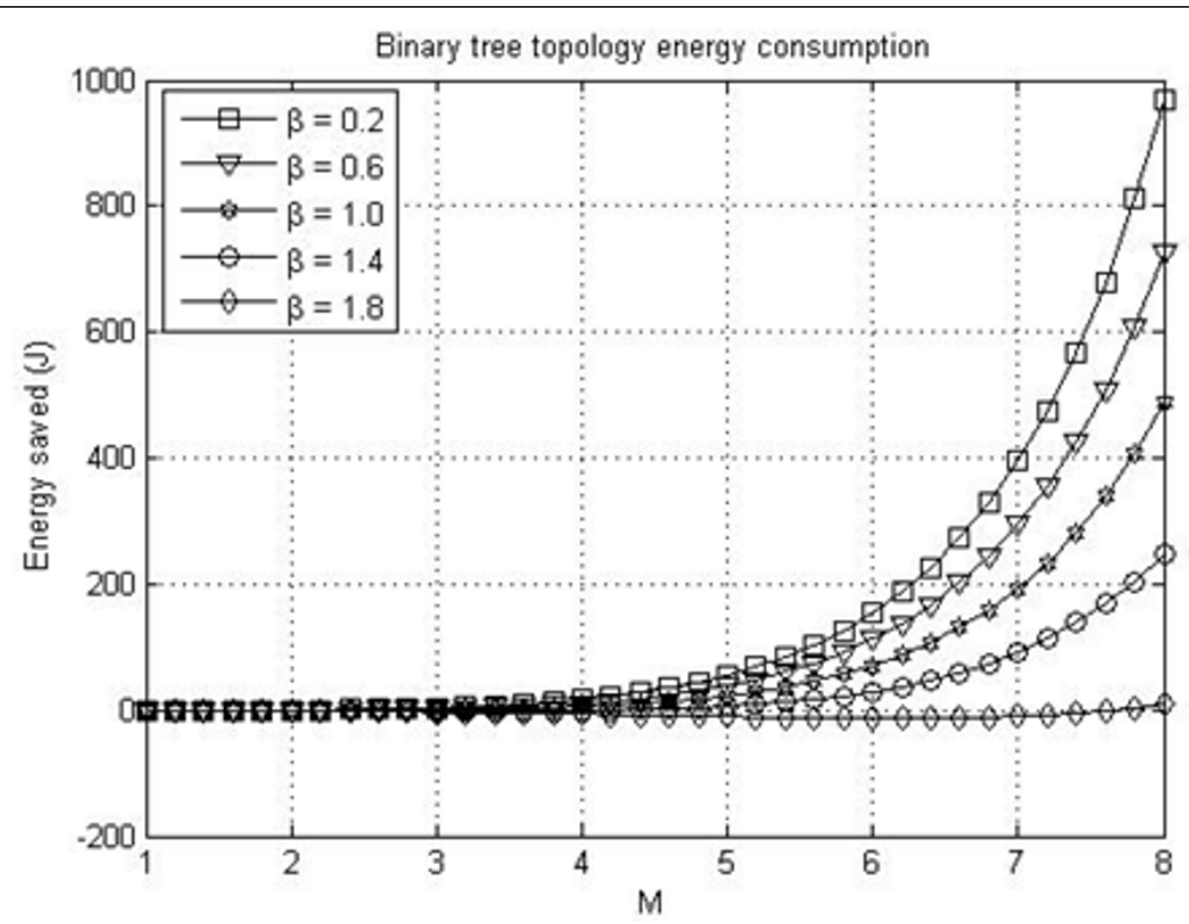

Figure 6 Energy saved under large-scale network

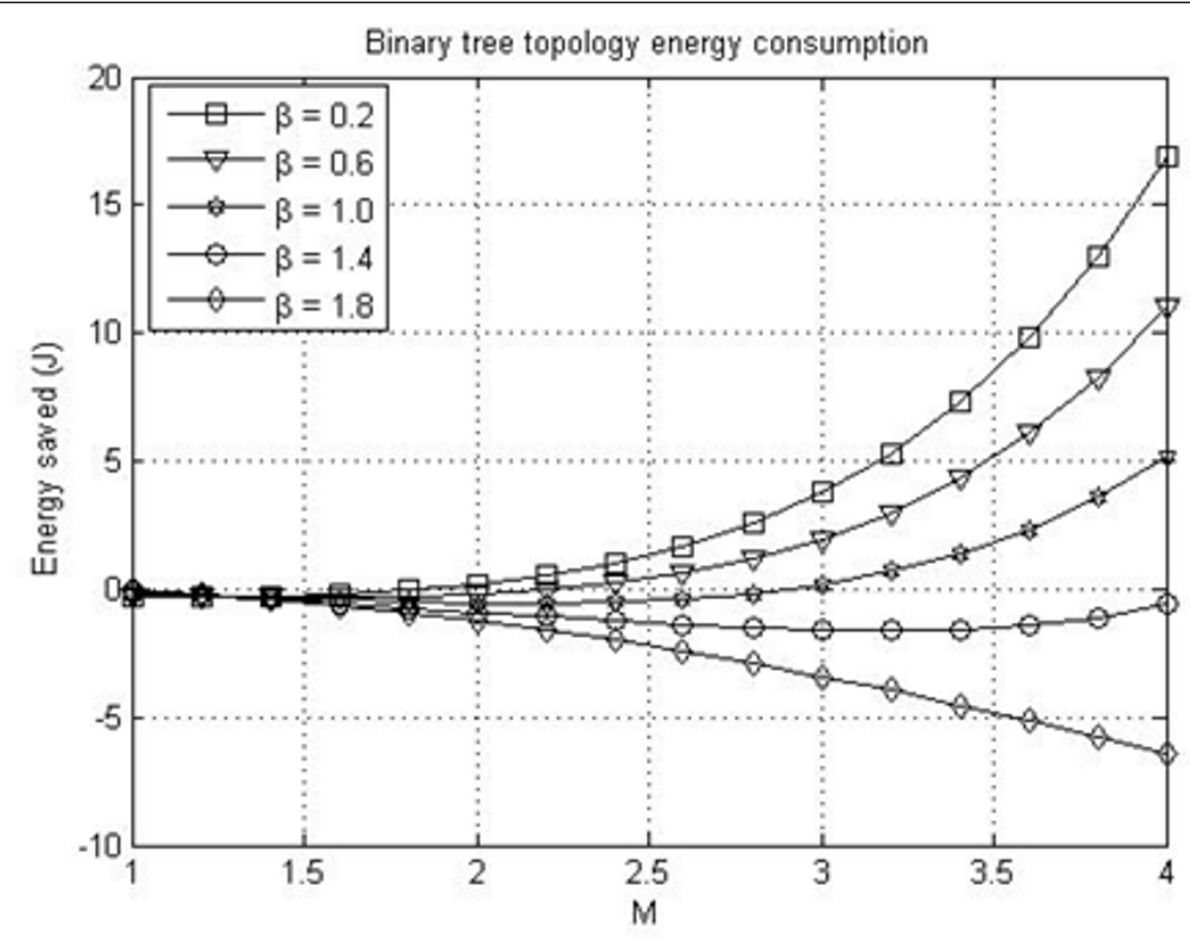

Figure 7 Energy saved under small-scale network. 


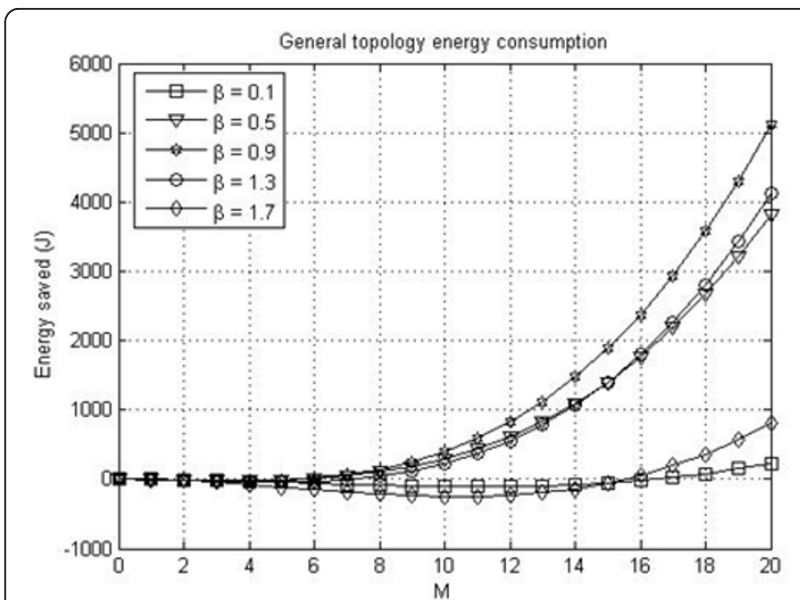

Figure 8 Energy saved under general topology

sector, but while the parameter $\lceil R / r\rceil$ maintains in small section such as lower than 20 , small $\beta$ as 0.1 cannot achieve high energy efficiency.

According to the simulation results shown above, we can further analyse meaningful guidelines in practical implementation of sensor networks. As illustrated in Figure 8 , we observe that even in suboptimal vertex angle such as 1.7 , the reverse process can still make the network more energy efficient than non-reverse one as the energy saving gain keeps increasing with respect to the ratio of coverage radius and transmission radius growth.

As depicted in Figures 10 and 11, after expanding the range of variable $\lceil R / r\rceil$, energy efficiency curves trends to be smooth and gradually approach the theory limitation of $E_{\text {efficient }}$. Thus, we can conclude that if the scale of a sector is configured properly, the reverse sector can save more energy than normal sector in terms of reducing hop count in the WSN. Through simple splitting and reverse process, energy efficiency can be enhanced by selecting proper divided vertex angle of each sector on the basis of network coverage and transmission capability of sensor nodes.

After the WSN has been properly divided into sectors, beacon cluster routing algorithm can enhance the energy conservation performance of each sensor nodes. As illustrated in Figure 12, we compare beacon cluster routing with two conventional routing algorithms for average dissipated energy in network. The network topology and division in the simulation is set as shown in Figure 4, sensor nodes in the network are divided into six different beacon clusters in beacon cluster routing scenario. However, for flooding and directed diffusion routing mechanisms, sector division is unnecessary, and multiple sinks collect sensor data at the same time.

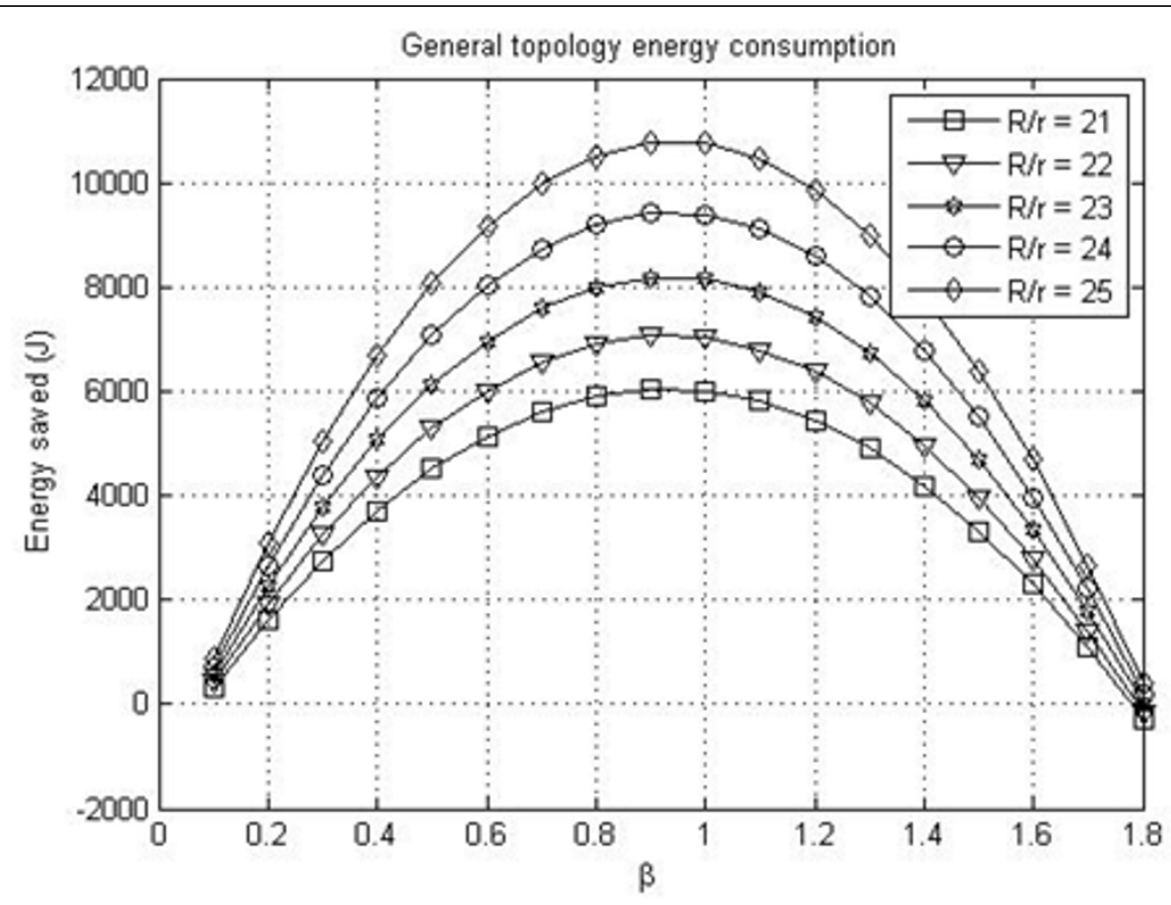

Figure 9 Maximum energy saved under general topology. 


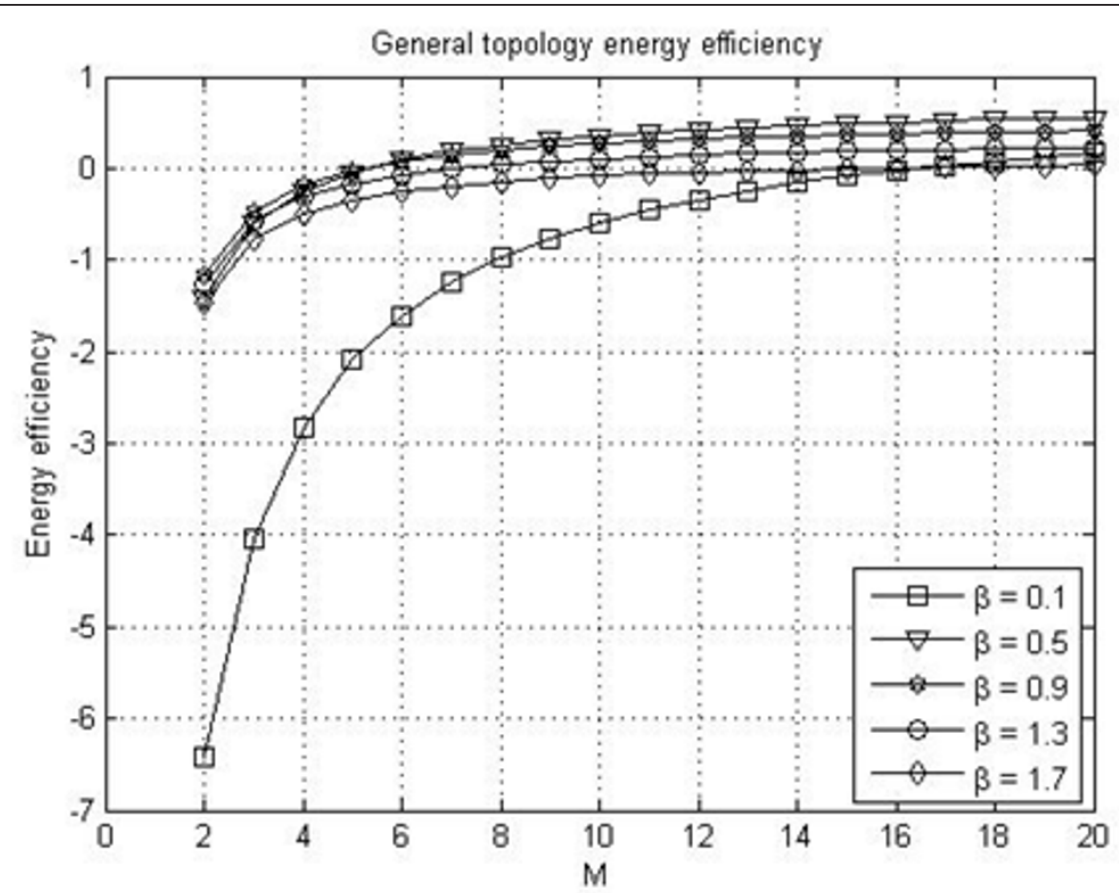

Figure 10 Energy efficiency under small-scale network

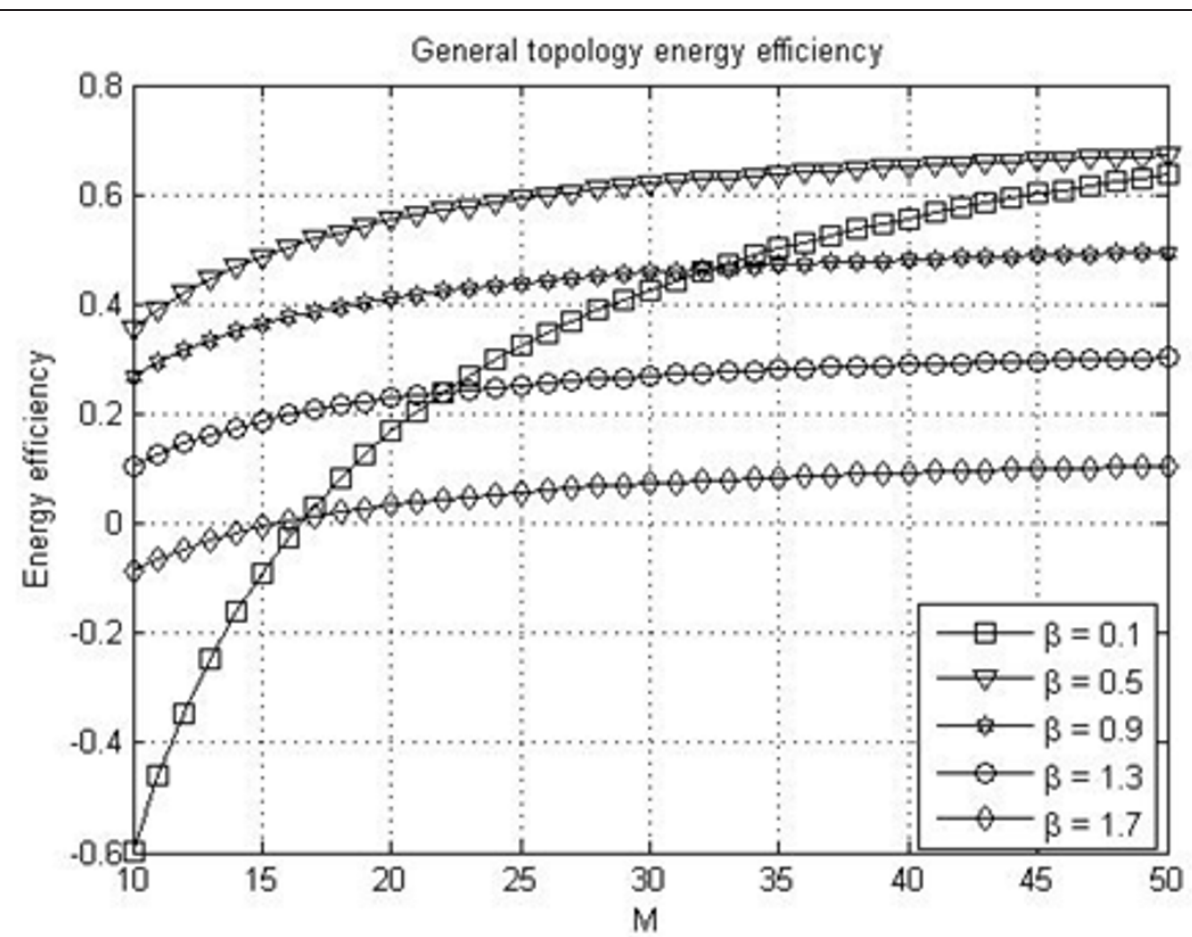

Figure 11 Energy efficiency under large-scale network. 


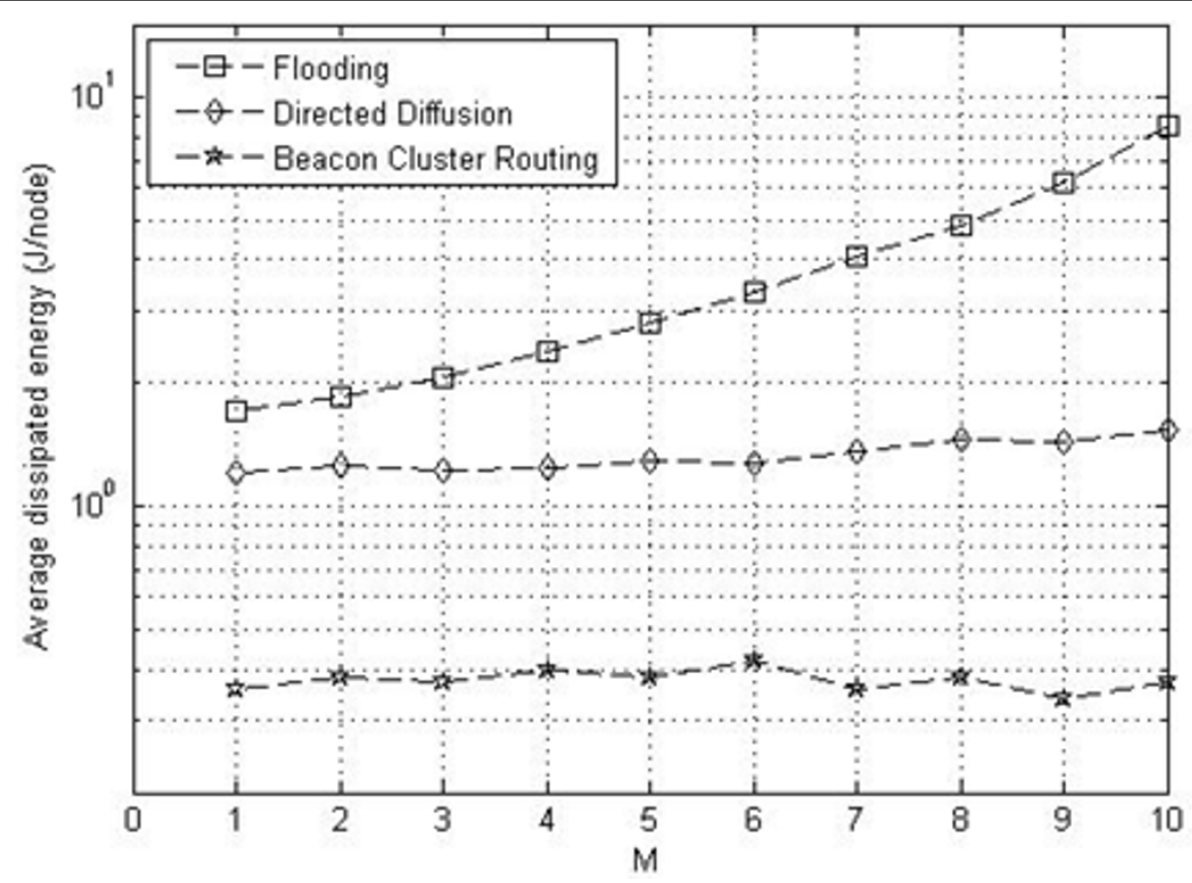

Figure 12 Average dissipated energy

Note that flooding is set as the benchmark for the performance comparison, and even diffusion performs much better than flooding. Beacon cluster routing outperforms the diffusion by more than $50 \%$ in terms of energy dissipation. Noticeably, beacon cluster routing dissipates less energy than these two conventional mechanisms since additional routing data exchanging procedures are replaced by local computation.

Figure 13 shows the delay performance comparison of three routing algorithms. As mentioned above, beacon cluster routing algorithm transfers the routing consumption into local computation consumption, each sensor nodes can deliver the packets immediately when the beacon broadcasting arrives. Thus, these three routing algorithms are separated with different order of magnitude in the delay performance comparison. With the increase of network scale, beacon cluster routing algorithm shows lower growth rate of network delay while the flooding obviously deteriorates network transmission efficiency. Furthermore, it is clearly shown that the beacon cluster routing is more efficient than the diffusion approach.

\section{Conclusions}

In this article, we proposed a beacon cluster based routing algorithm for the convergence of WSN and cellular network service applications. By virtue of the proposed solutions, it is possible to improve the energy and information collection efficiency in WSN. With the optimal network pre-implementation according to the sector splitting scheme, sub-optimization can be achieved in each divided beacon clusters. Accordingly, the performance of the whole network is optimized via optimal division during the routing procedure. More than 50\% of the energy consumption can be saved comparing with conventional network. Furthermore, it is possible to reduce the re-clustering communication overhead since the change of routes between the sensor nodes is minimized. Sensor network topology can adapt to the data collection pattern of real-time quantity and position of mobile gateways flexibly. Meanwhile the re-routing expenditure has been transformed to lower the distributed calculation consumption in the sensor network.

Analysis in this article is considered in the worst situations such as full binary tree while in practical scenarios, network structure can be simpler. This indicates that we have given the lower bound of the performance evaluation. With the optimization scheme we proposed in practical implementation, network performance can outperform our estimation in this paper. Trade-off between network scale and divided angle is helpful for network implementation. While we enlarge the scale of local sensor network via installing new sensor nodes for new requirement, divided angle of certain sectors should be re-configured in order to maintain the network work in optimal energy efficient status. Similarly, we may 


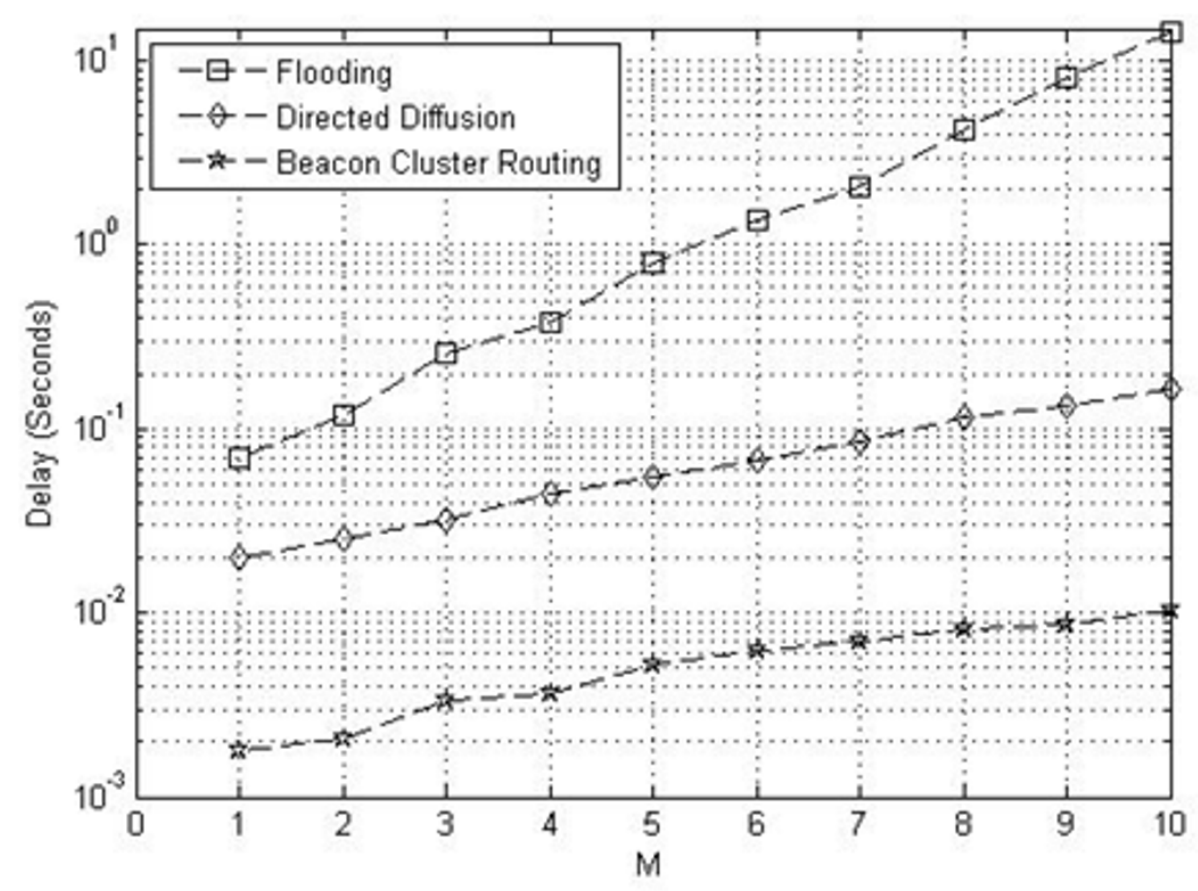

Figure 13 Delay performance comparison

reconfigure the density of the sensor nodes which in turn will decrease the transmission rage of single node to reach the goal of energy saving. Finally, we have presented simulation results to show the performance of the proposed algorithm. In addition, our proposed algorithms can be considered to further optimize in large scale wireless networks [20].

\section{Author details \\ 'Shanghai Institute of Micro-system and Information Technology (SIMIT), Chinese Academy of Sciences (CAS), Shanghai, China ${ }^{2}$ The State Key Laboratory of Integrated Services Networks (ISN), China ${ }^{3}$ Renesas Tele. Tech. (Beijing) Ltd., Shanghai, China ${ }^{4}$ Shanghai Advanced Research Institute, Chinese Academic of Science, Shanghai, China}

\section{Competing interests}

Partial content is patented by Renesas Tele. Tech. (Beijing) Ltd., Shanghai, China.

This work is supported by the National Science and Technology Major Project of the Ministry of Science and Technology of China under Grant No. 2009ZX03005-002-01, and National High-tech R\&D Program (863 Program) of China under Grant No. 2009AA012002.

This work is partially supported by the open research fund of the State Key Laboratory of Integrated Services Networks, Xidian University, China, and Natural Science Foundation of Shanghai under Grant 10ZR1436000, National High-Tech R\&D Program (863 Program) of China under Grant No.2011AA01A105.

Received: 15 July 2011 Accepted: 6 March 2012 Published: 6 March 2012

\section{References}

1. D Estrin, R Govindan, J Heidemann, S Kumar, Next century challenges: scalable coordination in sensor networks, in 5th Annual International Conference on Mobile Computing and Networking (MobiCom 1999), Seattle, WA, 1999, pp. 263-270

2. J Heidemann, F Silva, C Intanagonwiwat, R Govindan, D Estrin, D Ganesan, Building efficient wireless sensor networks with low-level naming. in Symposium on Operating Systems Principles. New York, 2001 35(5), 146-159

3. G Pottie, W Kaiser, Wireless integrated network sensors. Commun ACM. 43(5), 51-58 (2000). doi:10.1145/332833.332838

4. S Hedetniemi, A Liestman, A survey of gossiping and brocadcasting in communication networks. IEEE Network. 18(4), 319-349 (1988)

5. J Gao, L Guibas, J Hershberger, L Zhang, A Zhu, Geometric spanners for routing in mobile networks, in 2nd ACM International Symposium on Mobile Ad Hoc Networking and Computing (MobiHoc 2001), New York, 2001, pp. $45-55$

6. L Zhao, G Liu, J Chen, ZW Zhang, Flooding and directed diffusion routing algorithm in wireless sensor networks, in Ninth International Conference on Hybrid Intelligent Systems, Shenyang, 2009, pp. 235-239

7. WR Heinzelman, J Kulik, H Balakrishnan, Adaptive protocols for information dissemination in wireless sensor networks, in Proceedings of the ACM MobiCom'99, Seattle, WA, 1999, pp. 174-185

8. C Intanagonwiwat, R Govindan, D Estrin, Directd diffusion: a scalable and robust communication paradigm for sensor networks, MobiCom '00 Proceedings of the 6th annual international conference on Mobile computing and networking, New York, 2000, pp. 56-67

9. K Hwang, D Eom, Adaptive sink mobility management scheme for wireless sensor networks. Lecture Notes in Computer Science (LNCS). 4159 (2006)

10. G Wang, T Wang, W Jia, M Guo, HH Chen, M Guizani, Local update-based routing protocol in wireless sensor networks with mobile sinks, ICC '07. IEEE International Conference, Glasgow 3094-3099 (2007)

11. J Li, L Huang, G Wang, A novel clustering algorithm by using mobile gateways in densely deployed sensor networks. in ICIA 2008. International Conference on Information and Automation 1553-1559 (2008) 
12. K Langendoen, Medium Access Control in Wireless Networks, chapt. EnergyEfficient Medium Access Control, (Nova Science Publishers, 2008), pp. $535-560$

13. M Dohler, D Barthel, F Maraninchi, L Mounier, S Aubert, C Dugas, A Buhrig, F Paugnat, M Renaudin, A Duda, M Heusse, F Valois, The ARESA project: facilitating research, development and commercialization of WSNs, in 4th Annual IEEE Communications Society Conference on Sensor, Mesh and Ad Hoc Communications and Networks (SECON), San Diego, CA, USA, pp. 590-599 (June 2007)

14. C Dugas, Configuring and managing a large-scale monitoring network solving real world challenges for ultra-low powered and longrange wireless mesh networks. Int J Netw Manag. 15, 269-282 (2005). doi:10.1002/nem.573

15. A Bachir, M Dohler, T Watteyne, K Leung, Mac essentials for wireless sensor networks. IEEE Commun Surv Tutor. 12(2), 222-248 (2010)

16. P Gupta, PR Kumar, The capacity of wireless networks. IEEE Trans Inf Theory. 46(2), 388-404 (2000). doi:10.1109/18.825799

17. A El-Hoiydi, Aloha with preamble sampling for sporadic traffic in ad hoc wireless sensor networks, in ICC, vol. 5. New York, NY, IEEE, 2002, pp. 3418-3423

18. J Hill, D Culler, Mica: a wireless platform for deeply embedded networks IEEE Micro. 22(6), 12-24 (2002). doi:10.1109/MM.2002.1134340

19. W Ye, F Silva, J Heidemann, Ultra-low duty cycle MAC with scheduled channel polling, in 4th ACM Conference on Embedded Networked Sensor Systems (SenSys), Boulder, CO, ACM, pp. 321-334 (November 1-3 2006)

20. X Wang, W Huang, S Wang, J Zhang, C Hu, Delay and capacity tradeoff analysis for motioncast. IEEE/ACM Trans Netw. 19(5), 1354-1367 (2011)

\section{Submit your manuscript to a SpringerOpen ${ }^{\circ}$ journal and benefit from:}

- Convenient online submission

- Rigorous peer review

- Immediate publication on acceptance

- Open access: articles freely available online

- High visibility within the field

- Retaining the copyright to your article

Submit your next manuscript at $\gg$ springeropen.com 